\title{
Mikro-Nano-Integration in der Sensorik - Nanostrukturen als neue Option
}

Martin Hoffmann, Heike Bartsch, Michael Fischer, Stefan Hampl, Christoph Kremin, Steffen Leopold, Jens Müller, Tobias Polster, Mike Stubenrauch

Technische Universität Ilmenau, IMN MacroNano ${ }^{\circledR}$, Gustav-Kirchhoff-Str. 7, 98693 Ilmenau

\section{Zusammenfassung}

Für die Sensorik eröffnet der Einsatz von nanostrukturierten Materialien, die mit begrenztem Aufwand umsetzbar sind und für die keine kostspieligen und sehr aufwendigen Technologien benötigt werden, neue Perspektiven. Die Mikro-Nano-Integration, also die Integration von Nanostrukturen in Mikrosysteme, ist zumindest ein höchst interessantes Thema für zukünftige Sensoren.

Vorgestellt werden hier beispielhaft nanostrukturiertes Siliciumgras, das durch Selbstmaskierung in Trockenätzprozessen erzeugt werden kann, und nanokristallines Aluminiumnitrid, das mittels reaktiver Sputtertechnik erzeugt wird. Während Siliciumgras insbesondere für extrem große Oberflächen sorgt und auch als infrarot-optisches Interface von Bedeutung ist, sind Membranen aus nanokristallinem Aluminiumnitrid, das auch piezoelektrische Eigenschaften aufweist, extrem stabil und weisen eine sehr hohe thermische Leitfähigkeit auf.

\section{Siliciumgras als selbstmaskierende Nanostruktur}

Die ursprünglich eher unerwünschte, allenfalls zur Prozessoptimierung als Zwischenschritt verwandte Bildung von Siliciumnadeln in Trockenätzprozessen (als „Black Silicon“ bekannt) bietet viele noch nicht ausgenutzte Potenziale für die Sensorik. Der Effekt tritt sowohl bei klassischen, kontinuierlichen Reaktiven Ionenätzprozessen (RIE) auf (Black Silicon, [1]) als auch bei DRIE-Prozessen mit einem Wechsel zwischen Passivierung und Ätzen („Bosch-Prozess“). Erste Anwendungen des DRIE-basierten Prozesses in MEMS wurden in [2] aufgezeigt, z. B. ein Nano-Klettverschluss und die extreme Vergrößerung der Oberfläche durch Si-Gras in mikrofluidischen Kanälen. Der Einsatz von „Black Silicon“ ist auch für hochfeste hybride Substrate von Bedeutung [ 3 ], bei denen ein Siliciumwafer mit einem vorstrukturierten, noch „grünen“ LTCC-Keramikfolienstapel laminiert und anschließend im Verbund gesintert wird. Hierdurch wird eine bislang unerreichte Festigkeit des Verbundsubstrates erzielt, die auf die Nanostrukturierung der Siliciumoberfläche und die daran angepasste Zusammensetzung der Keramik zurückzuführen ist.
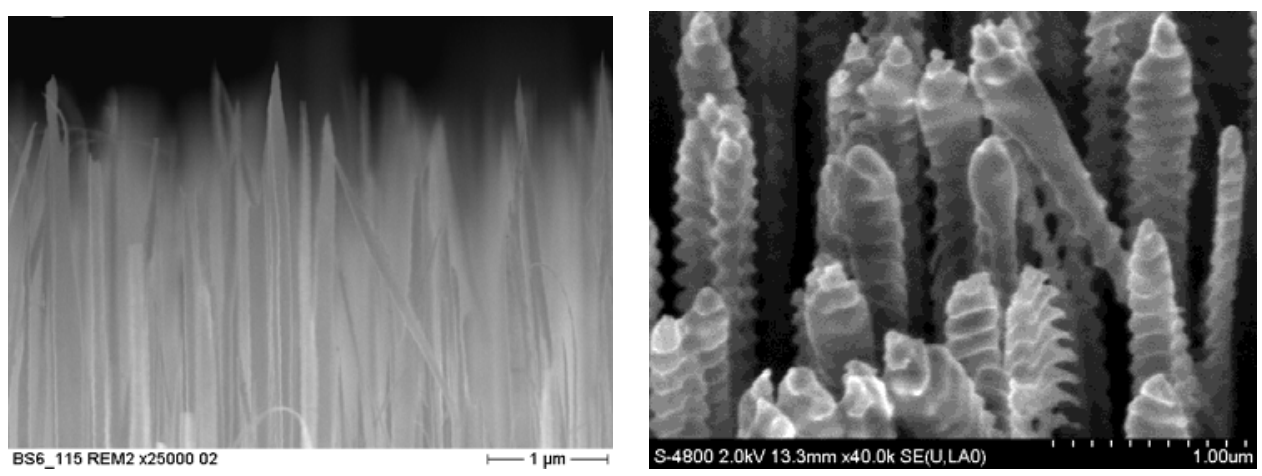

Bild 1 Unterschiedliche Si-Gras-Strukturen; die Nadeln bestehen aus einkristallinem Si und können durch die Prozessführung eingestellt werden.

Voraussetzung ist jedoch die reproduzierbare Herstellung von selbstorganisierten Nanostrukturen in und auf Mikrosystemen. Hier zeigte sich schnell, dass in der Literatur zwar zahlreiche Strategien zur Unterdrückung der Grasbildung beschrieben wurden, jedoch war unklar, wie die Bildung gezielt hervorgerufen und stabilisiert und der Prozess kontrolliert und gesteuert werden kann. Hier konnte in den letzten Jahren ein 
erheblicher Fortschritt erreicht werden. Insbesondere ist die Bildung der Maskierungskeime wichtig, damit der Start der Selbstmaskierung gleich in den ersten Ätzzyklen startet. Beim DRIE-basierten Prozess zeigt sich, dass diese Keime kohlenstoffhaltig, aber praktisch fluorfrei sind. Gleichzeitig zeigt das optische Emissionsspektrum des DRIE-Prozesses charakteristische Veränderungen, wenn die Selbstmaskierung stattfindet [4]. Bild 1 zeigt unterschiedliche Si-Gras-Strukturen, die durch gezielte Prozesssteuerung realisierbar sind. Damit sind wichtige Voraussetzungen für eine Prozesskontrolle gelegt, die für einen industriellen Einsatz zwingend erforderlich sind.

Nanostrukturen auf Silicium bieten insbesondere gute Perspektiven für infrarot-optische Sensoren, da sie eine effektive Reduzierung der Fresnel-Verluste an der Luft-Silicium-Grenzfläche durch Entspiegelung erlauben, ohne dass komplexe Schichtstapel notwendig sind. Darüber hinaus lassen sich fluidische Kanäle in Silicium so auch mit hydrophoben Beschichtungen versehen, die ähnlich wie der Lotuseffekt aufgebaut sind: zum einen ist die Oberfläche nanostrukturiert, so dass Flüssigkeiten nur sehr wenig Auflagefläche besitzen, und zum anderen sorgt der Herstellungsprozess im DRIE-Verfahren für eine dünne Passivierung der Oberfläche mit hydrophobem, fluorhaltigen Polymer.

Die Potenziale sind jedoch bei weitem noch nicht ausgeschöpft. Durch die Integration von nanostrukturierten Si-Oberflächen in Mikrosysteme können neue Funktionen in die Sensorik eingeführt werden. Mit den durch Ätzen erzeugten Nadeln steht damit ferner ein alternativer Prozess zu Si-Nanodrähten zur Verfügung, die als additiver Prozess die Herstellung von ebenfalls einkristallinen Nadeln auf Silicium erlauben. Am IMN MacroNano $^{\circledR}$ wurde bereits gezeigt, dass auch beide Prozesse (Si-Gras und Si-Nanodrähte) kombiniert werden können [5].

\section{Nanokristallines Aluminiumnitrid}

Nanokristallines Aluminiumnitrid (AlN) ist als piezoelektrische Funktionsschicht für Sensor-Anwendungen und in Filtern ein bekanntes Material. In aller Regel wird dabei vorrangig die piezoelektrische Eigenschaft des Materials genutzt, das als Dünnschicht mittels Reaktivem Sputtern auf Siliciumwafern erzeugt wird und dessen Herstellung weitgehend mit den Prozessen der Mikroelektronik kompatibel ist.

Darüber hinaus bietet AIN jedoch weitere, höchst interessante Eigenschaften für die Mikrosensorik. Es eignet sich insbesondere als hochstabiles Membranmaterial. Neben ebenen, geschlossenen Membranen können auch dreidimensionale und/oder durchbrochene, extrem dünne Sensormembranen erzeugt werden [6]. Die Abbildung 2 zeigt unterschiedliche Membranstrukturen aus 300-500 nm dicken AlN-Schichten, die mittels Si-DRIE auf zum Teil vorstrukturierten Si-Wafern abgeschieden und anschließend freigestellt wurden (Bild 3).

Da die Selektivität des DRIE-Tiefätz-Verfahrens für Silicium gegenüber AlN extrem hoch (nahezu unendlich) ist, können dünnste AlN-Membranen unterschiedlicher Form und Größe gleichzeitig im Trockenätzprozess freigestellt werden. Die sonst bei Si-basierten Membranschichten $\left(\mathrm{SiO}_{2}, \mathrm{SION}, \mathrm{Si}_{3} \mathrm{~N}_{4}\right)$ beobachtete Überätzung und damit Schwächung der Schichtdicke im Zentrum der Membran gerade bei größeren Öffnungen ist deshalb unproblematisch. Allerdings ist AlN nicht stabil in anisotropen SiÄtzlösungen wie Kalilauge $(\mathrm{KOH})$, es kann jedoch mit alkalischen Lösungen oder in Chlor-basierten Trockenätzprozessen strukturiert werden.
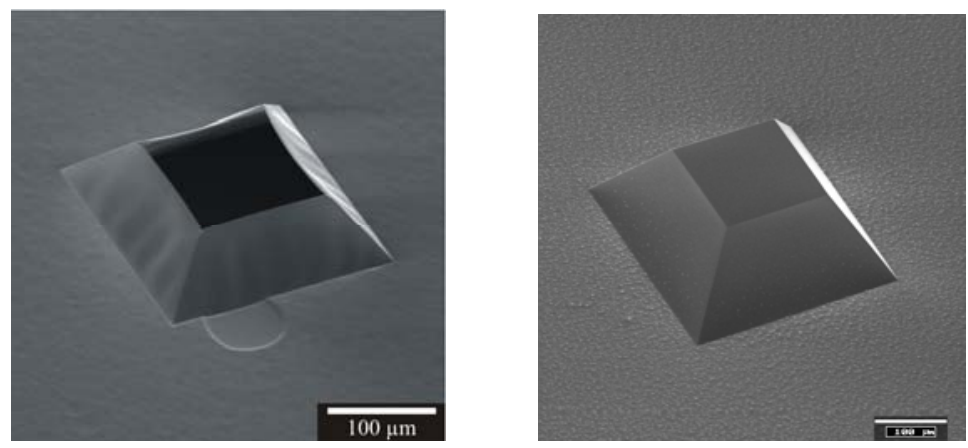

Bild 2 Dreidimensionale AlN-Membranen (500 nm dick): links: Druckspannungen führen zu einem Trichter; rechts: Spannungsfreie Pyramide aus piezoelektrischer AlN-Membran; 

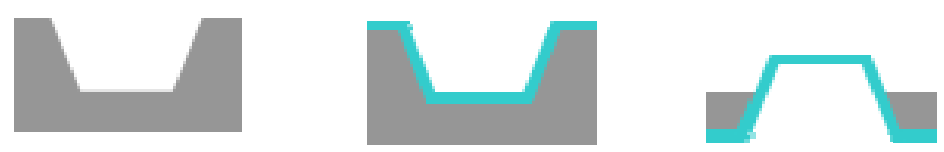

Bild 3 Die Abscheidung von AlN erfolgt in eine anisotrop geätzte Si-Pyramide, das Si wird dann von der Rückseite entfernt.

Die auch für die piezoelektrischen Eigenschaften verantwortliche Nanostruktur (c-Achsen der AlNKristallite senkrecht zur Oberfläche orientiert) sorgt für eine besonders stabile Membran, in der eine Rissausbreitung durch die zahlreichen Korngrenzen (Kristalldurchmesser im Bereich einiger Nanometer) stark behindert wird. Eine AlN-Membran von ca. $300 \mathrm{~nm}$ Schichtdicke ohne weitere Tragschichten hält bei einem Durchmesser von $1 \mathrm{~mm}$ einem Differenzdruck von rund $100 \mathrm{kPa}$ stand, bei $3 \mathrm{~mm}$ Durchmesser sind es ca. $50 \mathrm{kPa}$.

Ein weiteres Einsatzfeld sind Energy Harvester auf Basis von AlN, die zurzeit für ihre Eignung in minimalinvasiven Implantaten (am Beispiel eines Augendrucksensors) optimiert werden. Da AlN sowohl biokompatibel als auch CMOS-kompatibel ist, kann auch eine Integration in oder auf mikroelektronische Schaltungen vorgenommen werden. Schwerpunkt der Arbeiten ist zurzeit die Entwicklung möglichst effizienter Harvester für niedrige Frequenzen (einige 10 bis einige $100 \mathrm{~Hz}$, z. B. Sakkaden des menschlichen Auges) wie sie am oder im menschlichen Körper auftreten [7, 8].

\section{Literatur}

[1] H.V. Jansen, M.J. de Boer, S. Unnikrishnan, S. M.C. Louwerse, M.C. Elwenspoek, Black silicon method X: a review on high speed and selective plasma etching of silicon with profile control: an in-depth comparison between Bosch and cryostat DRIE processes as a roadmap to next generation equipment. Journal of micromechanics and microengineering, (2009) 19 (3). 033001

[2] M. Stubenrauch, M. Fischer, C. Kremin, S. Stoebenau, A. Albrecht, O. Nagel, Black silicon - new functionalities in microsystems, Journal of Micromechanics and Microengineering 16/6, S. 82-87

[3] M. Fischer, Michael; H. Bartsch de Torres, P. Pawlowski, R. Gade, S. Barth, M. Mach, M. Stubenrauch, M. Hoffmann, J. Müller, Silicon on ceramics - a new integration concept for silicon devices to LTCC, Journal of Microelectronics and Electronic Packaging (2009) 6, 1-5

[4] S. Leopold, C. Kremin, A. Ulbrich, S. Krischok, M. Hoffmann: Formation of silicon grass: Nanomasking by carbon clusters in cyclic deep reactive ion etching, Journal of Vacuum Science \& Technology B (Vol. 29, Issue 1)

[5] V. Cimalla, M. Stubenrauch, F. Weise, M. Fischer, K. Tonisch, M. Hoffmann, O. Ambacher, Suspended nanowire web, Applied Physics Letters 90, 101504 (2007)7

[6] T. Polster, M. Hoffmann, Aluminum nitride based 3D piezoelectric tactile sensor, Procedia Chemistry 1 (2009) 144147

[7] S. Hampl, V. Cimalla, T. Polster, M. Hoffmann, AlN-based piezoelectric bimorph microgenerator utilizing lowlevel non-resonant excitation, Proc. SPIE 8066, 80661E (2011)

[8] S. Hampl, D. Laqua, T. Polster, M. Hoffmann, AlN-basierte piezoelektrische Mikrogeneratoren zur Energieversorgung miniaturisierter Implantate, Tagungsunterlagen Mikrosystemtechnik-Kongress 2011, Darmstadt, 10.-12. Oktober 2011 